\title{
Glial cell line-derived neurotrophic factor (GDNF) attenuates the peripheral neuromuscular dysfunction without inhibiting the activation of spinal microglia/monocyte
}

Fei Xie $^{1+}$, Fan Zhang ${ }^{2+}$, Su Min ${ }^{1 *}$ (D) Jingyuan Chen ${ }^{1}$, Jun Yang ${ }^{1}$ and Xin Wang ${ }^{1}$

\begin{abstract}
Background: Peripheral neuromuscular dysfunctions were found in elderly individuals, and spinal microglia/ monocyte plays an important role on this process. This study aims to test whether the glial cell line-derived neurotrophic factor (GDNF) could attenuate age-related neuromuscular dysfunction by inhibiting the activation of spinal microglia/monocyte.

Methods: Male Sprague-Dawley rats were divided into an adult group and an aged group. The aged rats were intrathecally injected with normal saline (NS) and GDNF. All the rats were harvested 5 days after each injection. The muscular function was tested by compound muscle action potential, and the activation of microglia/monocyte was detected by immunofluorescence staining; cytokines were assayed by enzyme-linked immunosorbent assay; the expression level of GDNF and its known receptor GFR-a in the spinal cord, the expression level of neuregulin-1 (NRG-1) in the sciatic nerve, and the expression level of $\gamma$ - and a7- $\varepsilon$-nicotinic acetylcholine receptors in the tibialis anterior muscle were measured by western blotting.

Results: The activated microglia/monocyte was found in the aged rats compared to the adult rats. The aged rats showed a significant neuromuscular dysfunction and cytokine release as well as increased expression of $\gamma$ - and a7-nAChR. The protein expression of GDNF, GFR-a, and NRG-1 in the aged rats were significantly lower than that in the adult rats. However, the exogenous injection of GDNF could alleviate the neuromuscular dysfunction but not inhibit the activation of spinal microglia/monocyte. Furthermore, the levels of GFR-a and NRG-1 also increased after GDNF treatment
\end{abstract}

Conclusion: The GDNF could attenuate the age-related peripheral neuromuscular dysfunction without inhibiting the activation of microglia/monocyte in the spinal cord.

Keywords: Aging, Neuromuscular dysfunction, Microglia/monocyte, Spinal cord, Nicotinic acetylcholine receptors

* Correspondence: ms89011068@163.com

${ }^{\dagger}$ Equal contributors

${ }^{1}$ Department of Anesthesiology, the First Affiliated Hospital of Chongqing

Medical University, Friendship Road 1\#, Yuan Jia Gang, Chongqing 400016,

China

Full list of author information is available at the end of the article

(c) The Author(s). 2018 Open Access This article is distributed under the terms of the Creative Commons Attribution 4.0 International License (http://creativecommons.org/licenses/by/4.0/), which permits unrestricted use, distribution, and reproduction in any medium, provided you give appropriate credit to the original author(s) and the source, provide a link to the Creative Commons license, and indicate if changes were made. The Creative Commons Public Domain Dedication waiver (http://creativecommons.org/publicdomain/zero/1.0/) applies to the data made available in this article, unless otherwise stated. 


\section{Background}

Skeletal muscle dysfunction, which manifests as acquired muscle weakness and a reduced capacity to continue muscle contractions and other neurodegenerations, are observed in many elderly individuals [1, 2]. The demyelination of nerve has been considered the main mechanism for neuromuscular dysfunction [3]. Historically, studies have shown that the neuromuscular dysfunction was found in advanced age, and the demyelination of sciatic nerve is a factor [4]. Although the peripheral motor nerve plays an important role in muscular function, however, the central nervous system also contributes to muscular function in the elderly individuals.

The blood-spinal cord barrier (BSCB) is injured more often in the aging spinal cord than in the adult spinal cord, which was associated with increased tissue cytokine levels [5]. Microglia cell/monocyte, the resident innate immune cells in the central nervous system (CNS) that mediated neuroinflammation, are associated with many neurodegenerative disorders [6-8]. The microglia cell/monocyte exhibits greater immunoreactivity in the CNS of elderly individuals [9]. Other studies suggest that the aged microglia/monocyte cell becomes activated and leads to a status of "para-inflammation" [10-12], which is between basal homeostatic conditions and true inflammation. Even if this para-inflammation is slight, it is still detrimental to the CNS [13], and eventually aggravates peripheral motor nerve injury [14]. This indicates that peripheral nerve demyelination and muscular dysfunction may be a result of spinal cord inflammation [15].

Despite the proinflammatory effect, the microglia/ monocyte exerts protective effects by secreting neurotrophic factors after being activated [16-18]. It was shown that the glial cell line-derived neurotrophic factor (GDNF) is also a very potent trophic factor for spinal motoneurons [19] and central noradrenergic neurons [20]. Therefore, the GDNF raised great expectations as a potential therapeutic agent for the treatment of neurodegenerative diseases. The GDNF has a significant effect on the remyelination of neurons following spinal cord injury [21]. Recently, the effect of GDNF on the regeneration of the peripheral nerve has since been tested in some models of nerve injury [22]. Currently, however, it is unknown whether exogenous GDNF can modulate the function of spinal microglia cell/monocyte to alleviate the peripheral neuromuscular dysfunction in the advanced age.

In previous studies, three variants of nAChRs are observed in the post-junctional synapse. Normally, $\varepsilon$ nicotinic acetylcholine receptors (nAChRs) are confined to the neuromuscular junctions (NMJ), which are performed the muscle contraction. However, aging leads to the re-expression of $\alpha 7-n A C h R$ and $\gamma-n A C h R$ in skeletal muscles and demyelination of sciatic nerve [4], which are associated with peripheral neuromuscular dysfunction.

Based on the aforementioned phenomena and conclusions, in this study, we aimed to test whether the GDNF could attenuate the age-related neuromuscular dysfunction by inhibiting the activation of spinal microglia cell/ monocyte.

\section{Methods \\ Animals}

Male adult (4 months old, weight range: $220-235 \mathrm{~g}$ ) and male aged (16 months old, weight range: 460-478 g) Sprague-Dawley rats were purchased from Chongqing Medical University (Chongqing, China). All rats received humane care according to the Animal Ethics and Use Committee of Chongqing Medical University (GB149222001 and GB14927-2001). All rats were housed in a SPF room with the appropriate temperature and humidity, and the normal light/dark cycle). All the procedures were reviewed and approved by the Ethical Committee of Chongqing Medical University (No.2016-46), and followed with the animal care guidelines of the National Institutes of Health.

\section{Group assignments}

Ten male adult rats were assigned as the adult group, and 30 male aged rats were randomly divided into three groups as follows: (1) an aged group without any treatment ( $n=10)$; (2) an aged group with normal saline $(\mathrm{N}$. S) treated ( $\mathrm{n}=10) ;(3)$ an aged group with recombinant rat GDNF treated (Beijing Creanovo Biotechnology Co., Ltd., China, $\mathrm{n}=10)$. GDNF $(25 \mu \mathrm{g})$ or N.S was intrathecally injected into the subarachnoid space once daily injection for five days, respectively.

Intrathecal catheter implantation.

For intrathecal injection of inhibitors, the catheter implantation was performed as described previously [23, 24 with some modifications. After the animal was anesthetized by $0.5 \%$ sodium pentobarbital $(65 \mathrm{mg} / \mathrm{kg})$, a 2-cm longitudinal midline skin incision was made in the lumbar region (L4 - S1), and the intervertebral membrane between L5 and S1 was exposed. A 23-gauge needle was then used to puncture the membrane, and the PE-10 catheter was gently pushed into the L5-6 subarachnoid space at an angle of approximately $20^{\circ}$ to $30^{\circ}$ above the vertebral column to reach $\mathrm{L} 4$ at the lumbar enlargement. The inside catheter was then fixed to the muscle, and the other end was tunneled under the skin and pulled out of the cut at the neck. All incisions were closed with 4-0 silk and the neck end was sealed by heated paraffin. The rats were allowed to recover for 1 day before compound muscle action potential (CMAP) testing and intrathecal injections. The position of the catheter was checked postmortem. Animals that showed 
any abnormal neurological signs were excluded from experiments.

\section{Evaluation of neuromuscular function}

On the sixth day after injections, the rats were placed in the dorsal recumbent position after being anesthetized. The right sciatic nerve was exposed at the thigh with noncompliant silk. Stimulation electrodes (BL-420S Systems, Chengdu Techman Co., LTD, China) were attached to measure nerve-mediated contraction of the tibialis anterior muscle with several parameters (intensity $2 \mathrm{~V}$; duration $0.2 \mathrm{~ms}$; and frequency $1 \mathrm{~Hz}$ ). CMAP was recorded with a receiving electrode attached on the right tibialis anterior muscle [25]. The amplitude, latency time and duration of CMAP were analyzed with the BL-420S software. The motor conduction velocity was calculated as the distance of conduction/duration time. The temperature of each rat was kept at $36^{\circ}$ to $37^{\circ} \mathrm{C}$ by the heating light. A decrease of $\geq 20 \%$ of the lower limit of the normal CMAP amplitude could be considered as the neuromuscular dysfunction [26].

\section{Tissue preparation}

The rats were humanely euthanatized by deep anesthesia before the collection of tissues. The lumbar enlargement, sciatic nerves and the tibialis anterior muscle specimens of rats were collected in $4 \%$ paraformaldehyde for immunofluorescence staining or frozen with liquid nitrogen for western blot analysis.

\section{Cytokine measurements}

The lumbar enlargement samples were harvested immediately after decapitation. Samples were homogenized and were centrifuged at $2000 \mathrm{~g}$ for $15 \mathrm{~min}$ at $4{ }^{\circ} \mathrm{C}$ to separate the supernatant. The concentrations of TNF- $\alpha$ and IL- 6 were measured by enzyme-linked immunosorbent assay (ELISA) kits (the two kits were purchased from CUSABIO BIOTECH Co., Ltd., Wuhan, China), the procedures were followed the instructions.

\section{Immunofluorescence staining}

The deparaffinized spinal cord sections were permeabilized with $0.1 \%$ Triton X-100 and blocked with 5\% normal donkey serum (Beyotime Institute of Biotechnology, Beijing, China) for 30 minutes at $25{ }^{\circ} \mathrm{C}$. Then, the two kinds of primary antibodies including CD11b (ab8878, Abcam Ltd., dilution 1:200) and Iba-1 (ab178680, Abcam Ltd., dilution 1:200) were incubated with the spinal cord samples for $12 \mathrm{~h}$ at $4{ }^{\circ} \mathrm{C}$ separately. Negative controls without primary antibodies were included. After 3 washes with PBS, donkey anti-mouse Alexa-Fluor 594 (for CD11b) and donkey anti-rabbit Alexa-Fluor 488 (for Iba1) were used as secondary fluorescent probes for $30 \mathrm{~min}$ at $25{ }^{\circ} \mathrm{C}$. The sections were viewed by confocal microscopy (TCS SP2, Leica, Germany) and analyzed as individual images for $\mathrm{CD} 11 \mathrm{~b}$ and Iba-1 co-expression. The average optical density was analyzed in 10 randomly selected microscopic fields in five sections of each group at a 400 -fold magnification. CD11b was quantified as the average number of positive cells per field. A negative (no antibody) control was included. All the results were recorded by researchers who were blinded to the experimental group.

\section{Western blot analysis}

The proteins of the spinal cord, the sciatic nerves, and the skeletal muscles were prepared according to the previous studies [4]. In brief, the concentrations of proteins were determined by the BCA Protein assay kit (Beyotime, China). $50 \mu \mathrm{g}$ proteins were separated by $10 \%$ SDS-PAGE, and then were transferred to nitrocellulose membranes. The membranes were blocked with $5 \%$ skim milk in TBS, the membranes were incubated with primary antibodies at $4{ }^{\circ} \mathrm{C}$ : GDNF (sc-13,147, Santa Cruz Biotechnology, Santa Cruz, CA; dilution: 1:600), GFR- $\alpha 1$ (PRS1133, Sigma-Aldrich, St. Louis, MO; dilution: 1:500), $\gamma$-nAChR (sc-13,998, Santa Cruz Biotechnology; dilution: 1:500), $\alpha$ 7-nAChR (ab10096, Abcam; dilution: 1:800) and neuregulin-1 (NRG-1) (sc-28,916, Santa Cruz Biotechnology; dilution: 1:500). After washed with TBS containing $0.3 \%$ Tween-20, the membranes were incubated with corresponding secondary antibodies (dilution: 1:2, 000, Beyotime, China) for $1 \mathrm{~h}$ at $25^{\circ} \mathrm{C}$; the immunoreactive protein bands were visualized using ECL reagent (Beyotime, China), and the band intensity was performed with the Quantity One software. The relative expression levels of these proteins were expressed as values normalized to GAPDH or $\alpha$-tubulin.

\section{Statistical analysis}

All statistical analyses were performed using SPSS (version 17.0, SPSS). The results values are expressed as mean plus/minus standard deviation (SD). The data were analyzed using one-way analysis of variance to compare the within-group differences or the StudentNewman-Keuls q test to compare the between group differences. The differences in all data were considered statistically significant at a $P$-value $<0.05$.

\section{Results}

\section{Neuromuscular function}

The CMAP recorded of four groups is illustrated in Fig. 1a. In the adult group, the amplitude of CMAP was 16.23 $\pm 3.14 \mathrm{mV}$ (Fig. 1b). However, the amplitude of CMAP decreased significantly in the aged group, $(P<0.01$, Fig. 1b). The duration of CMAP in the aged group was significantly prolonged compared to that in the adult group $(P<0.01$, Fig. 1c). The nerve conduction velocity was significantly 


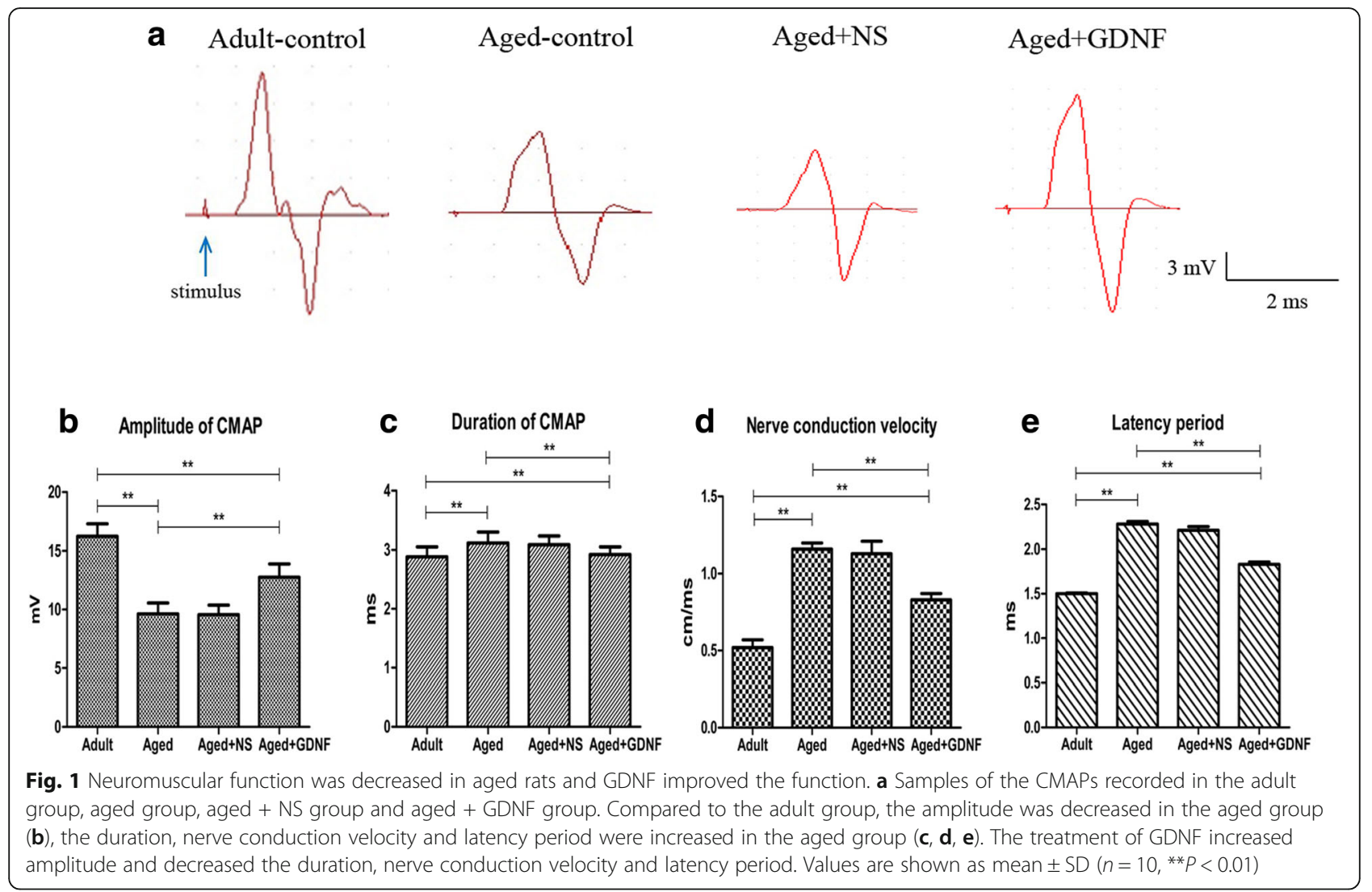

prolonged in the aged group compared to that in the adult group $(P<0.01$, Fig. $1 \mathrm{~d})$. The latency period was significantly prolonged in the aged group compared to that in the adult group $(P<0.01$, Fig. $1 \mathrm{e})$.

However, the amplitudes of CMAP were significantly increased in the GDNF-treated rats compared to that in the NS-treated rats $(P<0.05$, Fig. 1b). The durations of CMAP in the NS-treated rats were significantly longer than those in the GDNF-treated rats $(P<0.05$, Fig. $1 c)$. The nerve conduction velocity significantly decreased in the GDNF-treated rats compared to the NS-treated rats $(P<0.05$, Fig. 1d). Although the latency period was significantly increased in the NS-treated rats $(P<0.05$, Fig. 1e), it was significantly decreased in the GDNFtreated rats $(P<0.05)$.

\section{Cytokine plasma levels}

The concentrations of cytokine (TNF- $\alpha$ and IL-6) are presented in Fig. 2a and b. The cytokine level in the spinal cord, which was determined by ELISA, was low in the adult group. Compared to the adult group, the cytokine level was relatively increased in the aged group $(P<$ 0.01). However, the level of cytokines was still high in
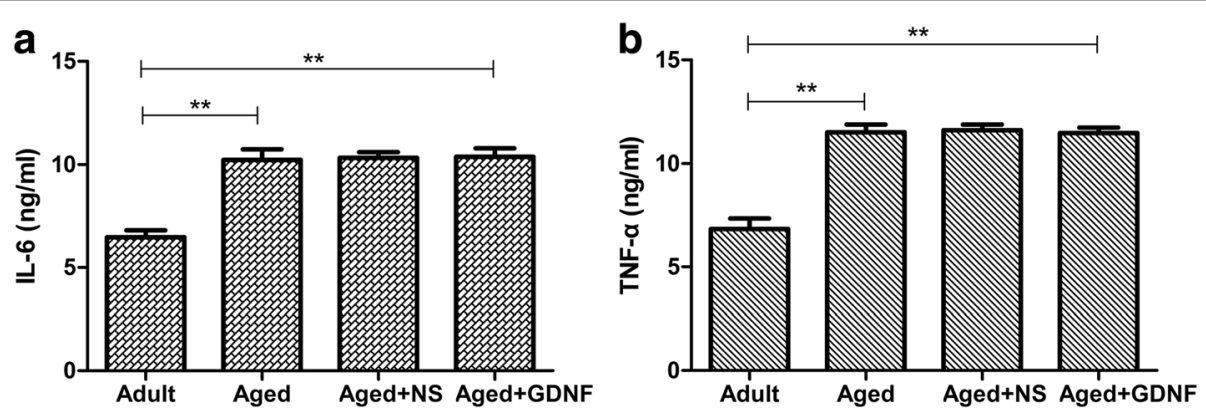

Fig. 2 Cytokines (TNF-a and IL-6) in the spinal cord for the four groups are shown (a and $\mathbf{b}$ ). Compared to the adult group, the cytokines were relatively increased in the aged group. The cytokines were still at high level after GDNF treatment compared to the aged group. Values are shown as mean $\pm \operatorname{SD}\left(n=10,{ }^{* *} P<0.01\right)$ 
the GDNF-treated group compared to that in the aged group $(P>0.05)$.

\section{Immunofluorescence staining}

The immunofluorescence staining results of CD11bpositive microglia/monocyte in the 4 groups are shown in Fig. 3a. Few or no CD11b-positive microglia cells/ monocytes were detected in the adult group. However, an increasing number of activated CD11b-positive microglia cells/monocytes were observed in the aged group. These activated microglia cells/monocytes exhibited relatively robust $\mathrm{CD} 11 \mathrm{~b}$ immunoreactivity compared to the adult group $(P<0.01$, Fig. 3b). GDNF could not inhibit the increase in CD11b-positive microglia cells/ monocytes of aged rats $(P<0.05$, Fig. $3 b)$, and microglia cells/monocytes still exhibited reactive morphology.

\section{Western blot results}

Western blotting showed that the protein expression of NRG-1, GDNF, and GFR- $\alpha 1$ were significantly decreased in the aged group $(P<0.01$, Fig. 4a). On the contrary, compared to the adult group, the protein expression levels of $\alpha 7-$ and $\gamma$-nAChR were increased in the aged group compared to those in the adult group $(P<0.01$, Fig. $4 \mathrm{~b})$.

The protein expressions of $\alpha 7-$ and $\gamma$-nAChR were decreased after treatment with GDNF $(P<0.01$, Fig. $4 a)$. In contrast, the protein expression levels of NRG-1 and GFR- $\alpha 1$ were significantly increased in the GDNF-

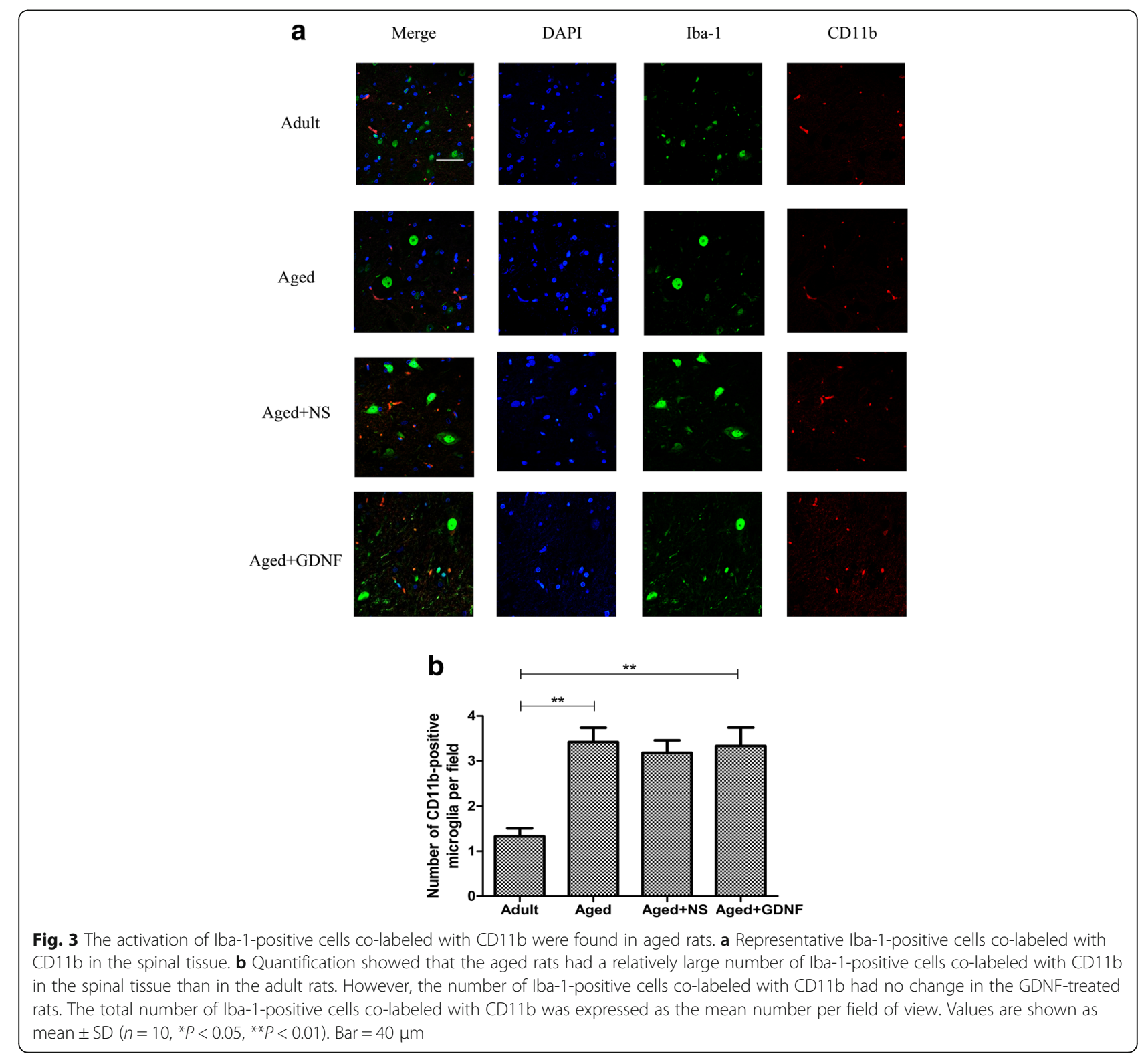




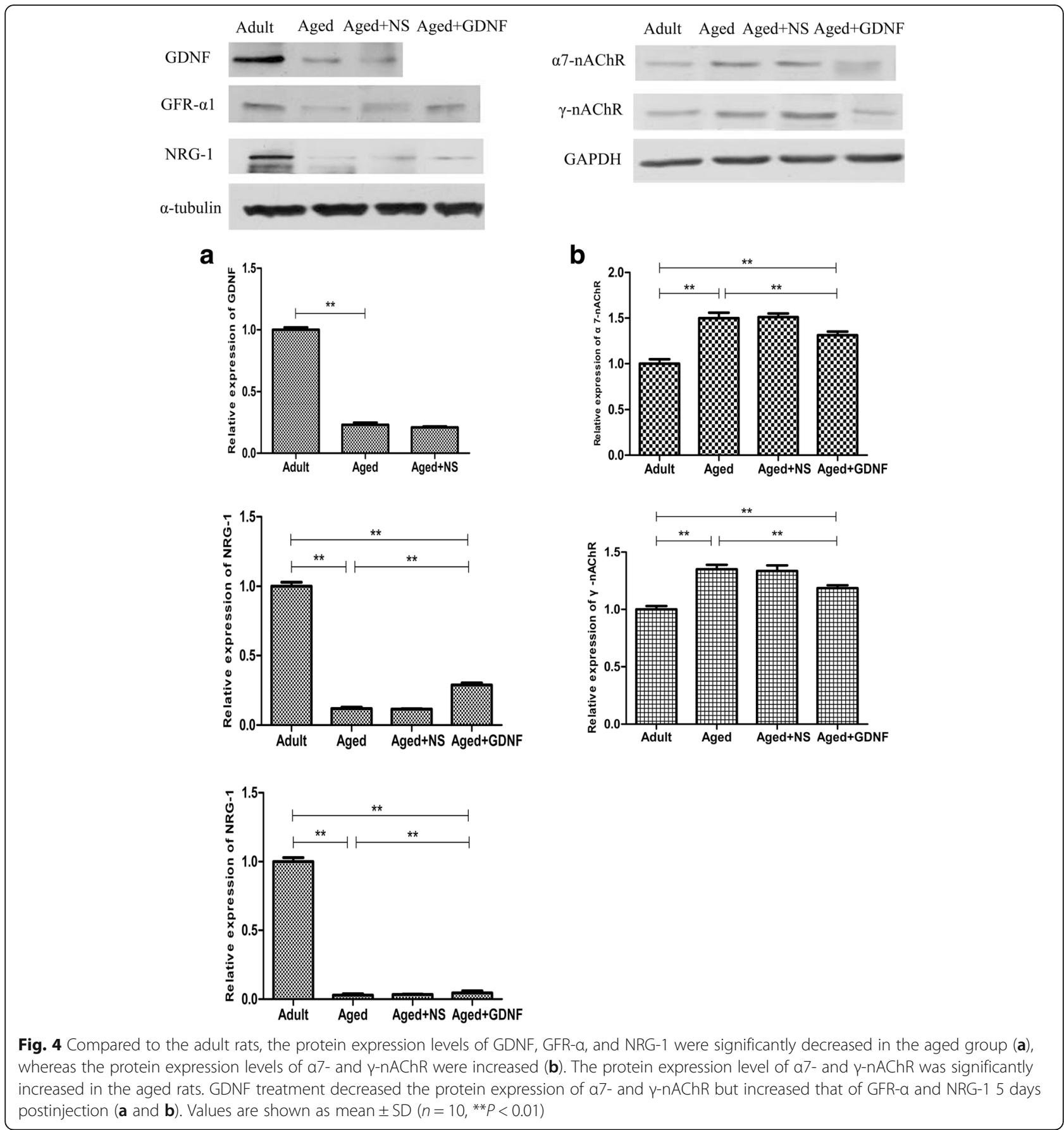

treated group compared to those in the NS-treated group $(P<0.01$, Fig. $4 \mathrm{~b})$.

\section{Discussion}

In this study, we showed that the GDNF attenuates the age-related neuromuscular dysfunction without inhibiting the activation of spinal microglia/monocyte. Our results showed that the CD11b-positive microglia/monocytes were activated and released a few cytokines in the aged spinal cord. Intrathecal injection of GDNF neither decreased the inflammatory response nor deactivated the microglia/monocytes in the aged spinal cord. According to our results, the insufficient level of GDNF could be one of the factors that induced neuromuscular dysfunction in aged rats.

The activation of microglia/monocytes results in chronic inflammation, a dysregulated or uncontrolled response to injury, and poorer recovery in advanced age [27]. The elevated basal cytokine level in the spinal cord may contribute to the higher activated state observed in 
these microglia/monocyte populations, especially with age, leading to a pro-inflammatory feedback loop that disrupts normal maintenance functions [5].

Previous studies also suggested that normal healthy aging was accompanied by neuroinflammation, which enhanced susceptibility to neurodegeneration [28, 29]. For example, multiple inflammatory markers increase with age in various brain and spinal cord regions of healthy rats, mice, and primates [5, 29-32]. In other words, the ability of senescent CNS to react to deleterious neurotoxic responses is decreased following injury, infection, or stress [33]. Furthermore, neuroimmune profiles of healthy aged CNS suggest that activation of microglia/monocytes [34] may contribute to neuromuscular disorders.

Although inflammation is associated with neuromuscular function in advanced age, the lack of neurotrophy could be another factor that could induce the dysfunction. GDNF could regulate the development and the differentiation of cells and neurons [35]. In addition, during some pathophysiological conditions, GDNF is upregulated in microglia/macrophages [36, 37]. Some studies showed that GDNF was secreted after the activation of microglia cells/monocytes in the CNS [38, 39]. However, in this study, we found that even if there was slight inflammation in the aged spinal cord, the neuromuscular dysfunction was significantly alleviated by exogenous injection of GDNF. Therefore, the insufficient level of GDNF in the aged spinal cord may be a reason for neuromuscular dysfunction.

Our previous studies demonstrated that aging induced upregulation of $\gamma$ - and $\alpha 7-n A C h R$ with downregulation of NRG-1 [4]. The expression of NRG-1 on the sciatic nerve may reflect the thickness of the myelin sheath [40]. In addition, NRG-1 had a regulatory role on the synthesis, expression, and stability of postsynaptic nAChRs through the MAP kinase signaling pathway, which were associated with the activation and expression of nAChRs [41, 42].

In this study, we found that the expression of GDNF was decreased in aged rats. Exogenous injection of GDNF could upregulate the expression of NRG-1 in the sciatic nerve and downregulate the expression of $\gamma$ - and $\alpha 7$ nAChR in skeletal muscles. The signaling mechanisms between GDNF and NRG-1 need more studies. Although the precise consequences of the upregulation of microglia/monocyte-derived GDNF in CNS inflammation have not been elucidated, enhancing the level of GDNF may contribute to a future therapeutic strategy for the treatment of inflammatory demyelinating diseases.

The spinal microglia was activated in advanced age, which was a factor of the neuroinflammation in the spinal cord. Previous studies had found that rat microglia express both Gfr- $\alpha 1$ and c-Ret, and our data supported these results. Interestingly, the GDNF-induced activation of c-Ret and subsequent phosphorylation of Erk $1 / 2$ has been previously reported in rat microglia [43, 44]. In this study, we demonstrated that GDNF was not able to inhibit aging-induced microglia activation, according to the expression and release of IL- 6 and TNF- $\alpha$. These results indicate GDNF might also have c-Retindependent functions in rat microglia.

Our study also had some limitations. The administration of GDNF is a short term treatment rather than a long term treatment. However, based on previous studies [45], we found that intrathecal injection of GDNF for 3 consecutive days could attenuate the mechanical allodynia and thermal hyperalgesia at $24 \mathrm{~h}$ after the first GDNF injection, and the analgesic effects continued for at least 3 days after the last injection. Thus, even though the microglia/monocytes had not been inactivated by GDNF, we proposed that the GDNF had enough effect after the last injection.

It had been reported that the microglia of female mice may express higher levels of cytokines [46]. These differences are associated with the secretion of testosterone surge in male mice after birth. Previous studies also proved that androgens can reduce the expression of proinflammatory cytokines in macrophages [46-48]. In addition, although there are no obvious differences in microglial gene expression between male and female, the function of microglial may be associated with sex differences. However, the male rats were examined in this study, so we should still consider the different microglial properties.

This study was performed using rat tissue and although the data had not suggested a regulatory potential of GDNF to downregulate microglia activation, it is still an open issue whether GDNF is able to directly inhibit aging-induced microglia activation.

\section{Conclusions}

Our findings demonstrated a vital role for GDNF in spinal microglia/monocytes in aged rat. The lack of GDNF in the aged spinal cord is associated with neuromuscular dysfunction, and exogenous injection of GDNF in the spinal cord improved the function recovery; however, GDNF could not inhibit the activation of microglia/monocytes.

\footnotetext{
Abbreviations

ANOVA: Analysis of variance; CMAP: Compound muscle action potential; CNS: Central nervous system; DAPI: 4',6-diamidino-2-phenylindole; ELISA: Enzyme-linked immunosorbent assay; GAPDH: Glyceraldehyde-3phosphate dehydrogenase; GDNF: Glial cell line-derived neurotrophic factor; MCV: Motor conduction velocity; MyD88: Myeloid differentiation factor 88; NF-kB: Nuclear factor-kappa B; NRG-1: Neuregulin-1; NS: Normal saline; PBS: Phosphate-buffered saline; PVDF: Polyvinylidene fluoride; $s$ nAChR: nicotinic acetylcholine receptor; SD rats: Sprague-Dawley rats; SDSPAGE: Sodium dodecyl sulfate polyacrylamidegel electrophoresis; SNK: Student-newman-keuls; TNF-a: Tumor necrosis factor alpha
} 


\section{Acknowledgements}

We thank the staffs at the Department of Laboratory Animal at Chongqing Medical University for data collection and the technical support from Fan Zhang at Department of Anesthesiology, the People's Hospital of Jianyang City, Chengdu, Sichuan, China.

\section{Funding}

This work was supported by the National Natural Science Foundation of China (No. 81471282), the Popularization Project of Health and Family Planning Commission of Sichuan Province (No. 17PJ228), and the Foundation of Postgraduate Innovation Project of Chongqing province, China (No. CYB16102). Sponsor's Role: the National Natural Science Foundation of China, the Popularization Project of Health and Family Planning Commission of Sichuan Province, and the Foundation of Postgraduate Innovation Project of Chongqing province had no role in the design or conduct of the study; collection, management, analysis, or interpretation of data; preparation, review, or approval of the manuscript; or the decision to submit the manuscript for publication. This study has not received funding/assistance from any commercial organization.

\section{Availability of data and materials}

All data generated or analyzed during this study are included in this published article.

\section{Authors' Contributions}

Study concept and design: FX, SM. Acquisition and analysis or interpretation of data: JYC, JY, FZ, XW. Drafting of manuscript: FX, SM. Critical revision of manuscript for important intellectual content: SM, FZ. Final approval of the version to be published: All authors have approved the final manuscript to be published.

\section{Competing interest}

The authors declare that they have no competing interests.

\section{Ethics approval and consent to participate}

The study protocol was approved by the Institutional Animal Care and Use Committee of Chongqing Medical University (No.2016-46), and was performed in accordance with the animal care guidelines of the National Institutes of Health. The rats were euthanatized by deep anesthesia before the collection of tissues.

\section{Publisher's Note}

Springer Nature remains neutral with regard to jurisdictional claims in published maps and institutional affiliations.

\section{Author details}

'Department of Anesthesiology, the First Affiliated Hospital of Chongqing Medical University, Friendship Road 1\#, Yuan Jia Gang, Chongqing 400016, China. ${ }^{2}$ Department of Anesthesiology, the People's Hospital of Jianyang City, Chengdu, Sichuan, China.

Received: 24 June 2017 Accepted: 30 April 2018 Published online: 09 May 2018

\section{References}

1. Strohacker K, Breslin WL, Carpenter KC, McFarlin BK. Aged mice have increased inflammatory monocyte concentration and altered expression of cell-surface functional receptors. J Biosci. 2012;37(1):55-62.

2. Compté $\mathrm{N}$, Zouaoui BK, Vanhaeverbeek $\mathrm{M}$, et al. Frailty in old age is associated with decreased interleukin-12/23 production in response to tolllike receptor ligation. PLoS One. 2013;8(6):e65325.

3. Deschenes MR, Kressin KA, Garratt RN, Leathrum CM, Shaffrey EC. Effects of exercise training on neuromuscular junction morphology and pre- to postsynaptic coupling in young and aged rats. Neuroscience. 2016;316:167-77.

4. Xie F, Min S, Liu L, Peng L, Hao X, Zhu X. Advanced age enhances the sepsis-induced up-regulation of the $\gamma$ - and a7-nicotinic acetylcholine receptors in different parts of the skeletal muscles. Arch Gerontol Geriatr. 2016;65:1-8

5. Ritzel RM, Patel AR, Pan S, et al. Age- and location-related changes in microglial function. Neurobiol Aging. 2015;36(6):2153-63.
6. Barcia C, Ros CM, Ros-Bernal F, et al. Persistent phagocytic characteristics of microglia in the substantia nigra of long-term parkinsonian macaques. J Neuroimmunol. 2013;261(1-2):60-6.

7. Wu J, Zhao Z, Sabirzhanov B, et al. Spinal cord injury causes brain inflammation associated with cognitive and affective changes: role of cell cycle pathways. J Neurosci. 2014;34(33):10989-1006.

8. Zhu HT, Bian C, Yuan JC, et al. Curcumin attenuates acute inflammatory injury by inhibiting the TLR4/MyD88/NF-kB signaling pathway in experimental traumatic brain injury. J Neuroinflammation. 2014;11:59.

9. Conde JR, Streit WJ. Microglia in the aging brain. J Neuropathol Exp Neurol. 2006;65(3):199-203.

10. Medzhitov R. Origin and physiological roles of inflammation. Nature. 2008; 454(7203):428-35.

11. Rao JS, Kellom M, Kim HW, Rapoport SI, Reese EA. Neuroinflammation and synaptic loss. Neurochem Res. 2012;37(5):903-10.

12. Wong WT. Microglial aging in the healthy CNS: phenotypes, drivers, and rejuvenation. Front Cell Neurosci. 2013;7:22.

13. Harry GJ. Microglia during development and aging. Pharmacol Ther. 2013 139(3):313-26

14. Casula M, lyer AM, Spliet WG, et al. Toll-like receptor signaling in amyotrophic lateral sclerosis spinal cord tissue. Neuroscience. 2011;179: 233-43.

15. Graber DJ, Hickey WF, Harris BT. Progressive changes in microglia and macrophages in spinal cord and peripheral nerve in the transgenic rat model of amyotrophic lateral sclerosis. J Neuroinflammation. 2010;7:8.

16. Loane DJ, Byrnes KR. Role of microglia in neurotrauma. Neurotherapeutics. 2010;7(4):366-77.

17. Ribes $\mathrm{S}$, Ebert $\mathrm{S}$, Regen $\mathrm{T}$, et al. Toll-like receptor stimulation enhances phagocytosis and intracellular killing of nonencapsulated and encapsulated Streptococcus pneumoniae by murine microglia. Infect Immun. 2010;78(2): 865-71.

18. Ribes S, Adam N, Schütze $\mathrm{S}$, et al. The nucleotide-binding oligomerization domain-containing-2 ligand muramyl dipeptide enhances phagocytosis and intracellular killing of Escherichia coli K1 by toll-like receptor agonists in microglial cells. J Neuroimmunol. 2012;252(1-2):16-23.

19. Henderson CE, Phillips HS, Pollock RA, et al. GDNF: a potent survival factor for motoneurons present in peripheral nerve and muscle. Science. 1994; 266(5187):1062-4.

20. Arenas E, Trupp M, Akerud P, Ibáñez CF. GDNF prevents degeneration and promotes the phenotype of brain noradrenergic neurons in vivo. Neuron. 1995;15(6):1465-73.

21. Blesch A, Tuszynski MH. Cellular GDNF delivery promotes growth of motor and dorsal column sensory axons after partial and complete spinal cord transections and induces remyelination. J Comp Neurol. 2003;467(3):403-17.

22. Ziv-Polat O, Shahar A, Levy I, et al. The role of neurotrophic factors conjugated to iron oxide nanoparticles in peripheral nerve regeneration: in vitro studies. Biomed Res Int. 2014:2014:267808.

23. Wu WP, Xu XJ, Hao JX. Chronic lumbar catheterization of the spinal subarachnoid space in mice. J Neurosci Methods. 2004;133(1-2):65-9.

24. Bai L, Zhai C, Han K, et al. Toll-like receptor 4-mediated nuclear factor-kB activation in spinal cord contributes to chronic morphine-induced analgesic tolerance and hyperalgesia in rats. Neurosci Bull. 2014;30(6):936-48.

25. Osuchowski MF, Teener J, Remick D. Noninvasive model of sciatic nerve conduction in healthy and septic mice: reliability and normative data. Muscle Nerve. 2009:40(4):610-6.

26. Van den Bergh PY, Piéret F. Electrodiagnostic criteria for acute and chronic inflammatory demyelinating polyradiculoneuropathy. Muscle Nerve. 2004; 29(4):565-74.

27. Schütze S, Ribes S, Kaufmann A, Manig A, Scheffel J, Redlich S, Bunkowski S, Hanisch UK, Brück W, Nau R. Higher mortality and impaired elimination of bacteria in aged mice after intracerebral infection with E. Coli are associated with an age-related decline of microglia and macrophage functions. Oncotarget. 2014:5:12573-92.

28. Franceschi C, Capri M, Monti D, et al. Inflammaging and anti-inflammaging: a systemic perspective on aging and longevity emerged from studies in humans. Mech Ageing Dev. 2007:128(1):92-105.

29. Jurgens HA, Johnson RW. Dysregulated neuronal-microglial cross-talk during aging, stress and inflammation. Exp Neurol. 2012;233(1):40-8.

30. Xie F, Zhang JC, Fu H, Chen J. Age-related decline of myelin proteins is highly correlated with activation of astrocytes and microglia in the rat CNS. Int J Mol Med. 2013;32(5):1021-8. 
31. Safaiyan S, Kannaiyan N, Snaidero N, et al. Age-related myelin degradation burdens the clearance function of microglia during aging. Nat Neurosci. 2016;19(8):995-8.

32. Niraula A, Sheridan JF, Godbout JP. Microglia priming with aging and stress. Neuropsychopharmacology. 2017;42:318-33.

33. Galbavy W, Kaczocha M, Puopolo M, Liu L, Rebecchi MJ. Neuroimmune and neuropathic responses of spinal cord and dorsal root ganglia in middle age. PLoS One. 2015;10(8):e0134394.

34. Sierra A, Gottfried-Blackmore AC, McEwen BS, Bulloch K. Microglia derived from aging mice exhibit an altered inflammatory profile. Glia. 2007;55(4): $412-24$

35. Nagano M, Suzuki H. Quantitative analyses of expression of GDNF and neurotrophins during postnatal development in rat skeletal muscles. Neurosci Res. 2003;45(4):391-9.

36. Ikeda T, Koo H, Xia YX, Ikenoue T, Choi BH. Bimodal upregulation of glial cell line-derived neurotrophic factor (GDNF) in the neonatal rat brain following ischemic/hypoxic injury. Int J Dev Neurosci. 2002;20(7):555-62.

37. Hashimoto M, Nitta A, Fukumitsu H, Nomoto H, Shen L, Furukawa S. Involvement of glial cell line-derived neurotrophic factor in activation processes of rodent macrophages. J Neurosci Res. 2005;79(4):476-87.

38. Chandel NS, Trzyna WC, McClintock DS, Schumacker PT. Role of oxidants in NF-kappa B activation and TNF-alpha gene transcription induced by hypoxia and endotoxin. J Immunol. 2000;165(2):1013-21.

39. Suh SJ, Chung TW, Son MJ, et al. The naturally occurring biflavonoid, ochnaflavone, inhibits LPS-induced iNOS expression, which is mediated by ERK1/2 via NF-kappaB regulation, in RAW264.7 cells. Arch Biochem Biophys. 2006;447(2):136-46.

40. Tsutsumi K, Yamashita Y, Ushio S, et al. Oxaliplatin induces hypomyelination and reduced neuregulin 1 expression in the rat sciatic nerve. Neurosci Res. 2014;80:86-90

41. Fromm L, Rhode M. Neuregulin-1 induces expression of Egr-1 and activates acetylcholine receptor transcription through an Egr-1-binding site. J Mol Biol. 2004:339(3):483-94.

42. Kim HG, Lee CK, Cho SM, et al. Neuregulin 1 up-regulates the expression of nicotinic acetylcholine receptors through the ErbB2/ErbB3-PI3K-MAPK signaling cascade in adult autonomic ganglion neurons. J Neurochem. 2013;124(4):502-13.

43. Boscia F, Esposito CL, Di CA, de Franciscis V, Annunziato L, Cerchia L. GDNF selectively induces microglial activation and neuronal survival in CA1/CA3 hippocampal regions exposed to NMDA insult through ret/ERK signalling. PLoS One. 2009:4:e6486.

44. Wang X, Min S, Xie F, Yang J, Li L, Chen J. Glial cell-derived neurotrophic factor alleviates sepsis-induced neuromuscular dysfunction by decreasing the expression of $\gamma$ - and a7-nicotinic acetylcholine receptors in an experimental rat model of neuromyopathy. Biochem Biophys Res Commun. 2018;496:260-6.

45. Kimura M, Sakai A, Sakamoto A, Suzuki H. Glial cell line-derived neurotrophic factor-mediated enhancement of noradrenergic descending inhibition in the locus coeruleus exerts prolonged analgesia in neuropathic pain. $\mathrm{Br} J$ Pharmacol. 2015;172:2469-78.

46. Crain JM, Nikodemova M, Watters JJ. Microglia express distinct M1 and M2 phenotypic markers in the postnatal and adult central nervous system in male and female mice. J Neurosci Res. 2013;91:1143-51.

47. Brown CM, Xu Q, Okhubo N, Vitek MP, Colton CA. Androgen-mediated immune function is altered by the apolipoprotein $\mathrm{E}$ gene. Endocrinology. 2007;148:3383-90.

48. Vignozzi L, Morelli A, Sarchielli E, Comeglio P, Filippi S, Cellai I, Maneschi E, Serni S, Gacci M, Carini M, et al. Testosterone protects from metabolic syndrome-associated prostate inflammation: an experimental study in rabbit. J Endocrinol. 2012;212:71-84.

Ready to submit your research? Choose BMC and benefit from:

- fast, convenient online submission

- thorough peer review by experienced researchers in your field

- rapid publication on acceptance

- support for research data, including large and complex data types

- gold Open Access which fosters wider collaboration and increased citations

- maximum visibility for your research: over $100 \mathrm{M}$ website views per year

At BMC, research is always in progress.

Learn more biomedcentral.com/submissions 